
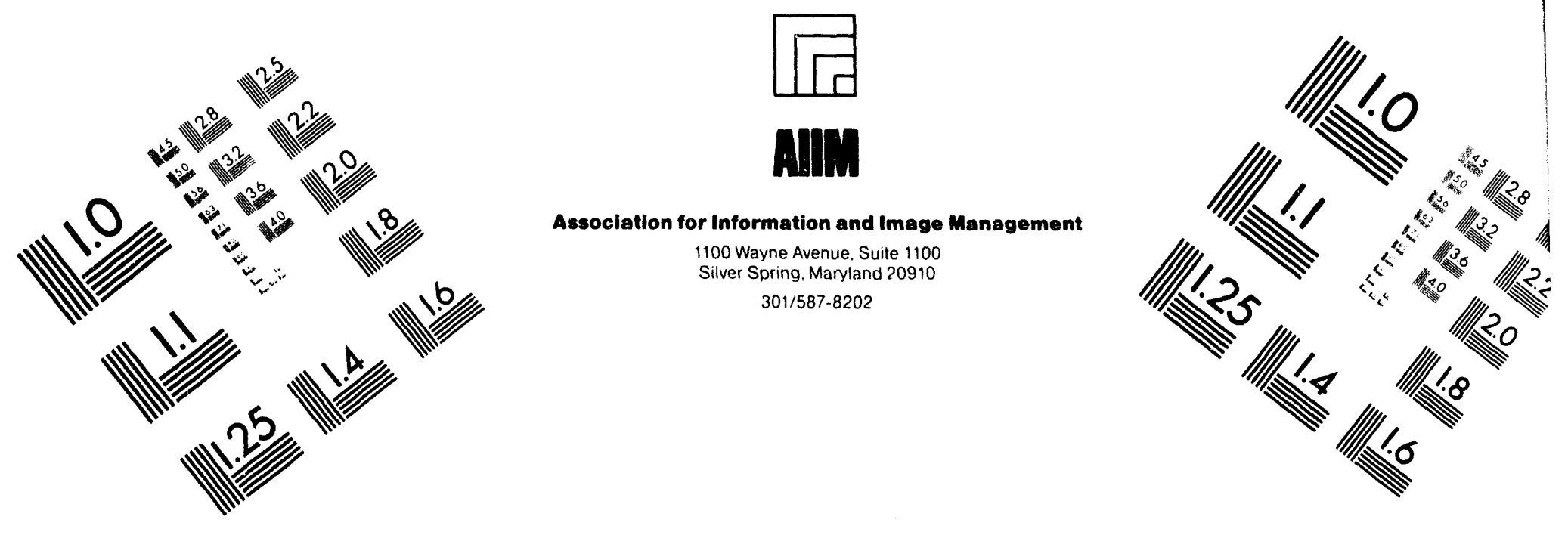

\title{
Centimeter
}

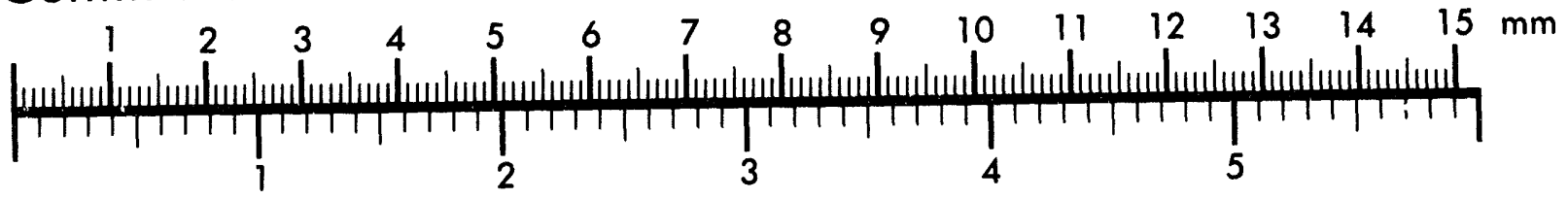
Inches

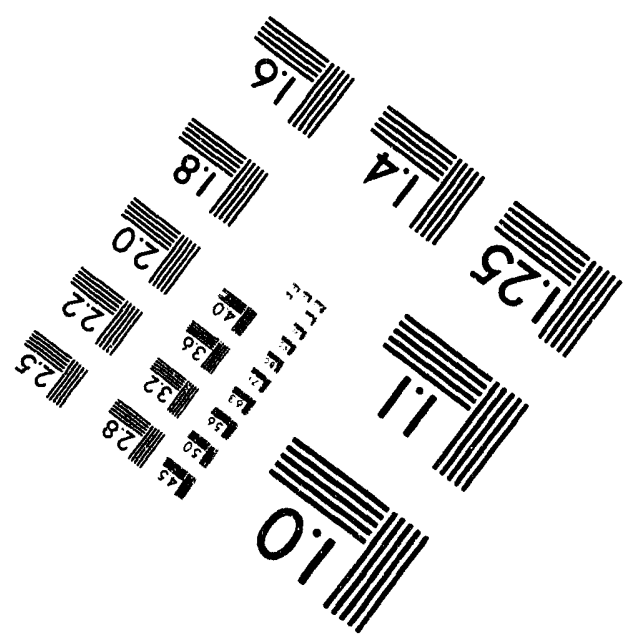

MAN! IFACTURED TO AIIM STANDARDS

BY APPLIED IMAGE. INC.

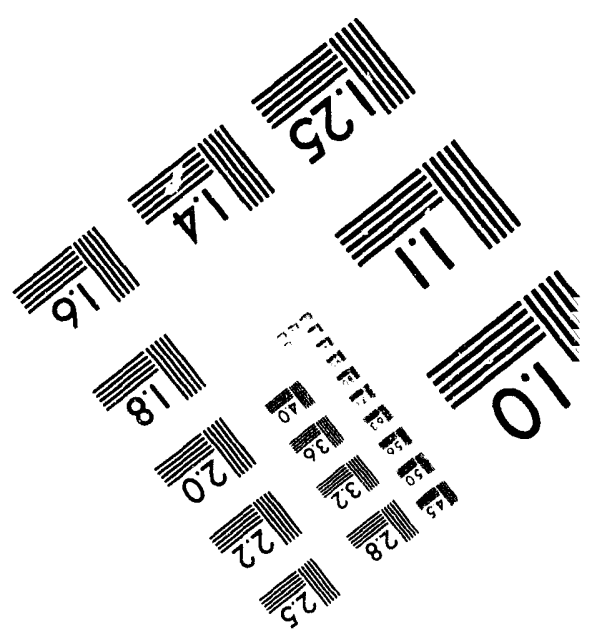



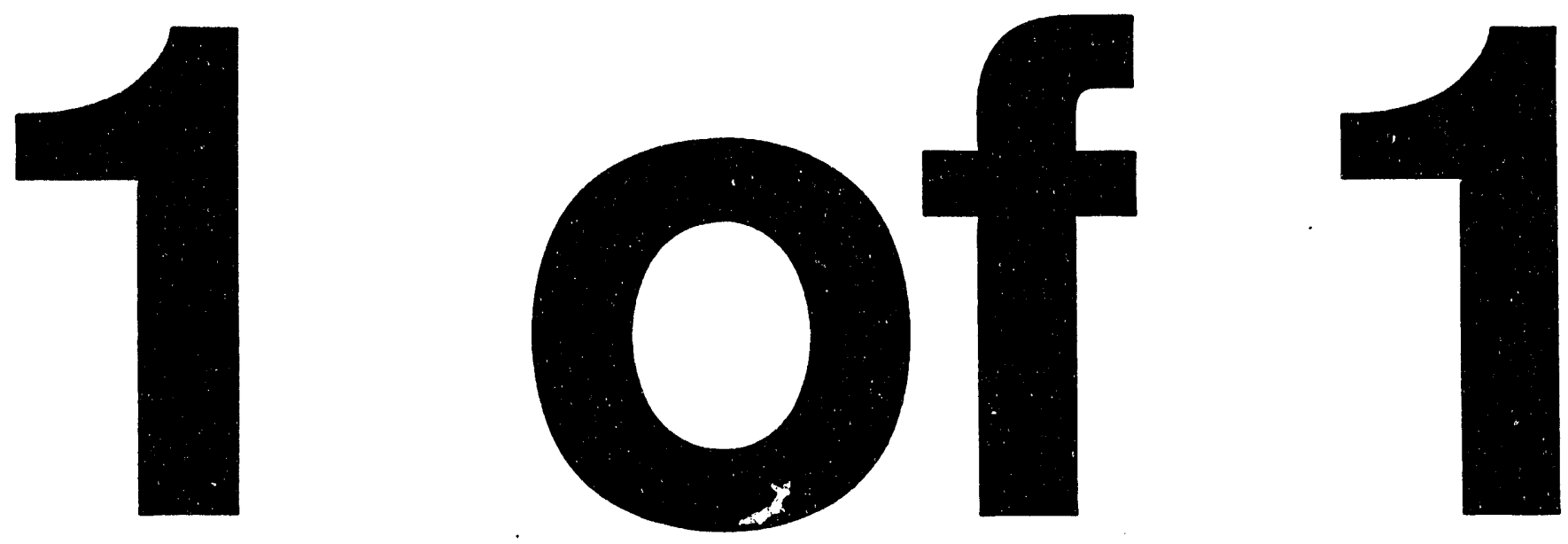


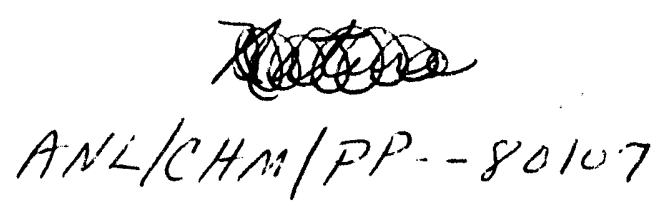

\section{A PHOTOPHYSICAL CONTROL MECHANISM FOR ZEAXANTHIN-ASSOCIATED RADIATIONLESS ENERGY DISSIPATION IN PHOTOSYNTHESIS}

\section{Harry A. Frank, ${ }^{1}$ Agnes Cua, ${ }^{1}$ Andrew Young, ${ }^{2}$ David Gosztola ${ }^{3}$ \& Michael R. Wasielewski ${ }^{3}$}

${ }^{1}$ Department of Chemistry, University of Connecticut, Storrs, CT 06269-3060, USA; ${ }^{2}$ School of Biological and Earth Sciences, Liverpool John Moores University, Byrom Street, Liverpool L3 3AF, UK; ${ }^{3}$ Chemistry Division, Argonne National Laboratory, Argonne, IL 60439, USA

Understanding the manner in which excess solar energy is dissipated by photosynthetic membranes under conditions of high light stress is a major problem facing researchers studying photosynthesis. 1 Light energy absorbed but not used to drive the photosynthetic light reactions must be deactivated by plants in a manner that does not lead to the photodestruction of the photosynthetic apparatus. The dissipation of this excess light energy can be measured in higher plants and algae by the extent to which chlorophyll fluorescence originating from the light-harvesting pigment-protein complexes is quenched under different chemical and physical conditions. ${ }^{1}$ It has long been known that a specific group of carotenoids known as xanthophylls are associated with these light-harvesting complexes. ${ }^{2}$ A correlation has been established between one such xanthophyll, zeaxanthin, and the magnitude of the fluorescence quenching. 1,3,4 The nature of the specific molecular mechanism by which zeaxanihin is able to quench chlorophyll fluorescence and thereby dissipate excess absorbed excitation energy has eluded researchers for years. ${ }^{1}$ Herein, we report femtosecond time-resolved, fast-transient optical spectroscopic analyses of three important xanthophylls. This work strongly supports the notion that the enzymatic reactions that interconvert these xanthophylls act as a kind of "molecular gear shift" controlling whether the molecules function as light-harvesting pigments performing forward energy transfer or as fluorescence quenchers performing reverse energy transfer.

Three specific xanthophylls, violaxanthin, antheraxanthin, and zeaxanthin, typically found in the light-harvesting apparatus of higher plants and algae, are interconverted into one another in the chloroplast membrane in a process referred to as the "xanthophyll cycle". 5 In this cycle, exposure of the photosynthetic system to light levels exceeding those that can be used for photosynthesis induces the enzymatic de-epoxidation of violaxanthin to antheraxanthin and zeaxanthin. Limiting light levels or dark adaptation of the photosynthetic membranes reverses the process, reforming violaxanthin by epoxidation of zeaxanthin and antheraxanthin. See Fig. 1. 
A strong correlation between the zeaxanthin content of leaves or chloroplasts and energy-dependent quenching of chlorophyll fluorescence $(\mathrm{qE})$ has recently been established. 1 In leaves, $\mathrm{qE}$ accounts for the majority of all non-photochemical quenching ( $\mathrm{qN}$ ) observed, with zeaxanthin accounting for $60-70 \%$ of this process. The remaining $\mathrm{qE}$ appears to be independent of zeaxanthin formation, although other xanthophylls may be involved. Some of the excess photon energy that is absorbed by chlorophyll, rather than being transferred to the reaction center, is dissipated by a form of radiationless energy transfer that involves zeaxanthin in the light-harvesting apparatus, but not the other xanthophylls. Interestingly, the effect is reversible. The fluorescence quenching typically relaxes within a few minutes upon returning the leaves to darkness. This finding is strongly suggestive of zeaxanthin playing a protective role of dissipating excess light energy which may otherwise lead to damage to the photosynthetic apparatus.

Previously, carotenoids (xanthophylls are one class of carotenoids possessing oxygencontaining functional groups) were thought to play only two roles in photosynthesis - that of light-harvesting pigment and triplet state quencher. ${ }^{6}$ As light-harvesting pigments, carotenoids supplement the light-capturing ability of chlorophyll by absorbing in regions of the visible spectrum where chlorophyll does not absorb. Carotenoids then transfer this energy to chlorophyll, which is capable of carrying out the primary photochemistry of photosynthesis. As triplet state quenchers, carotenoids are able to protect the photosynthetic apparatus by quenching highly reactive chlorophyll triplet states as soon as they are formed. Both of these processes, light-harvesting and triplet state quencher, require energy transfer. In the former process, carotenoids transfer singlet state energy to chlorophylls. In the latter process, carotenoids accept triplet state energy from chlorophylls. The two roles are thought to be made energetically $\mathrm{I}$ ossible by the fact that carotenoids, in general, have singlet states that are higher in energy and triplet states that are lower in energy than those of chlorophyll. Recent fluorescence studies on carotenoids have revealed, however, that their singlet states are not as high in energy as previously thought. ${ }^{7,8}$ Herein, it is demonstrated that a low-lying singlet state is responsible for the zeaxanthin-mediated fluorescence quenching of chlorophyll.

The singlet state energy diagram of carotenoids consists of at least two excited states that account for many of their photochemical properties. ${ }^{6}$ These are denoted ${ }^{1}{ }^{1} \mathrm{~B}_{\mathrm{u}}$ and $2^{1} \mathrm{~A}_{\mathrm{g}}$ according to the idealızed group theoretical $\left(\mathrm{C}_{2} \mathrm{~h}\right.$ point group) designations. The ground state designation is $1^{1} \mathrm{Ag}_{\mathrm{g}}$. The easily observed, prominent, visible, absorption spectrum that characterizes all carotenoids, is attributable to a strongly allowed transition from the ground $1^{1} A_{g}$ state to the $1^{1} B_{u}$ state. Lying below the $1^{1} B_{u}$ state is the $2^{1} A_{g}$ state into which absorption from the ground state is symmetry forbidden. The low oscillator strength of this ${ }^{1} A_{g} \rightarrow 2^{1} A_{g}$ transition has made the determinations of the precise energies of the $2^{1} A_{g}$ 
states of carotenoids somewhat difficult. Upon absorption of light, rapid internal conversion (on the order of a iew hundred femtoseconds) occurs from the $1^{1} \mathrm{~B}_{\mathrm{u}}$ to the $2^{1} \mathrm{~A}_{\mathrm{g}}$ state. ${ }^{9}$ This process represents a primary route of energy dissipation of carotenoids and of energy transfer to chlorophylls. ${ }^{6}$ Thus, the energies of the $2^{1} \mathrm{~A}_{\mathrm{g}}$ states are extremely important in assessing the energetic feasibility of energy transfer from carotenoids to chlorophyll. Fluorescence studies have revealed the $2^{1} \mathrm{~A}_{\mathrm{g}}$ state energies of some shorter carotenoids. However, the longer chain carotenoids ( $>9$ carbon-carbon double bonds) tend not to fluoresce from the $2^{1} \mathrm{~A}_{\mathrm{g}}$ state, but rather exhibit fluorescence originating from the $1^{1} \mathrm{~B}_{\mathrm{u}}$ level. 8

One method of obtaining the $2^{1} \mathrm{~A}_{\mathrm{g}}$ energies of the long chain carotenoids is by extrapolation from the shorter molecules whose $2^{1} \mathrm{~A}_{\mathrm{g}}$ energies have been determined by fluorescence spectroscopy. 8 This type of analysis is somewhat uncertain since only very few short chain length carotenoids have been characterized, and variations in their structures, primarily due to differences in their attached functional groups, make correlations between long and short carotenoids difficult. Recently, we have used picosecond time-resolved transient absorption spectroscopy to measure the $2^{1} \mathrm{~A}_{\mathrm{g}}$ state energy of the carotenoid, spheroidene. ${ }^{10}$ In these experiments, the $2^{1} \mathrm{~A} g$ state lifetimes of spheroidene and three alltrans analogs of spheroidene, 3,4,7,8-tetrahydrospheroidene, 3,4,5,6-tetrahydrospheroidene, and 3,4-dihydrospheroidene were measured. These molecules are structurally identical except for having $\pi$-electron conjugations that systematically increase from 7 to 10 carbon-carbon double bonds. Because the three spheroidene analogs exhibit fluorescence from their $2^{1} \mathrm{~A}_{\mathrm{g}}$ states, ${ }^{8}$ and therefore, determine the energies of those states, a measurement of the $2^{1} \mathrm{~A}_{\mathrm{g}}$ state lifetimes of these molecules "calibrates" the effect different $2^{1} \mathrm{~A}_{\mathrm{g}}$ state energies of carotenoids have on their decay dynamics. Furthermore, the application of the "energy gap law" for nonradiative transitions, ${ }^{11}$ which describes the magnitude of change in internal conversion rate constant with changes in state energy, makes it possible to determine the energies of the $2^{1} \mathrm{~A}_{\mathrm{g}}$ states of molecules such as spheroidene, which do not exhibit fluorescence from their $2^{1} \mathrm{~A}_{\mathrm{g}}$ levels. 10 The use of the energy gap law for carotenoids is valid because the quantum yields of carotenoid fluorescence are typically very low, leading to a dominance of the nonradiative pathway of singlet state decay from their $2^{1} \mathrm{~A}_{\mathrm{g}}$ states. The $2^{1} \mathrm{~A}_{\mathrm{g}}$ state energies of carotenoids can be determined by fitting the generalized energy gap law equation

$$
\mathrm{k}_{\mathrm{ic}}=c \cdot \exp \left(-\gamma \Delta \mathrm{E} / \hbar \omega_{\mathrm{M}}\right)
$$

to the dynamics data. 10 In this equation $\mathrm{k}_{\mathrm{ic}}$ is the internal conversion rate constant, for carotenoids, very well approximated by $1 / \tau$ because $k_{\text {ic }}$ for polyenes is much larger than the radiative rate constant, $\mathrm{k}_{\mathrm{f}} \sim 10^{9} \mathrm{~s}^{-1} . \Delta \mathrm{E}$ is the $2^{1} \mathrm{~A}_{\mathrm{g}}-1^{1} \mathrm{~A}_{\mathrm{g}}$ energy difference, $c$ is a 
constant, $\hbar \omega_{M}$ is the energy of the high frequency "acceptor" modes, and $\gamma$ can be related to the relative displacement of the potential surfaces in the two electronic states. The best fit to the data on the series of spheroidene molecules yielded a value of $8.98 \times 10^{-4} \mathrm{~cm}$ for $\gamma / \hbar \omega_{M}$ and $3.71 \times 10^{16}$ for $c$. The values of these parameters are applicable to all carotenoids because, in general, $C-C$ stretching vibrations, $\hbar \omega_{M} \sim 1300-1600 \mathrm{~cm}^{-1}$, have been shown to govern internal conversion from $2^{1} \mathrm{Ag} \cdot 12$ Also, it is likely that all $2^{1} \mathrm{Ag}_{\mathrm{g}}$ states of carotenoids are characterized by a reasonably narrow range of $\gamma$ values. 11

In the present work, the energies of the $2^{1} \mathrm{Ag}$ excited states of violaxanthin, antheraxanthin and zeaxanthin were determined. Similar to the experiments on the spheroidene analogs, a $300 \mathrm{fs}, 420 \mathrm{~nm}$ laser pulse was used to excite the xanthophylls into their $1^{1} \mathrm{~B}_{\mathrm{u}}$ states. After subsequent relaxation to the $2^{1} A_{g}$ state, the ${ }^{1} A_{g} \rightarrow S_{n}$ electronic absorption bands in the region $510-540 \mathrm{~nm}$ were probed and observed to decay with single exponential kinetics: $\tau=23.9 \mathrm{ps}$ for violaxanthin, $\tau=14.4 \mathrm{ps}$ for antheraxanthin and $\tau=9.0 \mathrm{ps}$ for zeaxanthin. See Fig. 2. These data used in conjunction with the energy-gap law expression given above yields $2^{1} \mathrm{~A}_{\mathrm{g}}$ state energies of $15,200 \mathrm{~cm}^{-1}, 14,700 \mathrm{~cm}^{-1}$ and $14,200 \mathrm{~cm}^{-1}$ for violaxanthin, antheraxanthin and zeaxanthin, respectively.

The most compelling feature of these data is revealed when one compares the energies of the $2^{1} \mathrm{Ag}$ states of the xanthophylls with that of the lowest excited singlet state of chlorophyll a in the light-harvesting complexes of Photosystem II (LHCII). See Fig. 3. In this complex, chlorophyll a exhibits its red-most maximum absorption $\left(\mathrm{Q}_{\mathrm{y}}\right.$ transition) at $\sim 680 \mathrm{~nm}$. This translates into a value of $14,700 \mathrm{~cm}^{-1}$ for the $S_{1}$ excited singlet state energy of chlorophyll which lies below that for violaxanthin but higher than the value for zeaxanthin. These data demonstrate that it is energetically possible for the $2^{1} A_{g}$ state of zeaxanthin to quench the excited state singlet of chlorophyll a in vivo. It is not energetically possible for violaxanthin to perform this same function. Conversely, it is energetically possible for violaxanthin to act as a light-harvesting pigment, absorbing light energy and transferring it from its $2^{1} \mathrm{~A}_{\mathrm{g}}$ state to chlorophyll a. It is not energetically possible for zeaxanthin to transfer energy via its $2^{1} \mathrm{~A}_{\mathrm{g}}$ state to chlorophyll a. Zeaxanthin may still act as a light-harvesting pigment, however, by transferring energy from its $1^{1} \mathrm{~B}_{\mathrm{u}}$ state to chlorophyll. Thus, the enzymatic de-epoxidation/epoxidation reactions of the xanthophyll cycle appear to be acting as a sort of "molecular gear shift". Under conditions of low light, with violaxanthin as the primary xanthophyll present, the carotenoid acts as a light-harvesting pigment transferring energy forward from its $2^{1} A_{g}$ state to the $S_{1}$ state of chlorophyll. Under high light conditions, de-epoxidation of violaxanthin to zeaxanthin occurs, and the system shifts into reverse, transferring the $S_{1}$ energy of chlorophyll back to the $2^{1} A_{g}$ state of zeaxanthin. The possibility of reverse energy transfer from chlorophyll to the $2^{1} \mathrm{Ag}_{\mathrm{g}}$ state of carotenoids was 
suggested by Demmig-Adams ${ }^{1}$ in response to the findings of Snyder et al. ${ }^{7}$ that the $2^{1} \mathrm{~A}_{\mathrm{g}}$ states of carotenoids may be much lower than previously thought. ${ }^{13}$ The low-lying nature of the $2^{1} \mathrm{~A}_{\mathrm{g}}$ states of carotenoids was confirmed by the work of DeCoster et al. ${ }^{8}$ These ideas have also been elaborated on recently by Owens et al. 14 and are supported by two-photon spectroscopic experiments from this group. The data presented here demonstrate conclusively the energetic feasibility of this "molecular gear shift" model and provide a reasonable explanation for the correlation of the presence of zeaxanthin with the occurrence of nonphotochemical quenching of chlorophyll fluorescence. It is important to note that these data do not exclude other controlling factors such as aggregation being important as well. 15 For energy transfer to occur into and out of the carotenoid $2^{1} \mathrm{~A}_{\mathrm{g}}$ state, which has a low oscillator strength for a transition from the ground state, the exchange mechanism is likely to be operative. ${ }^{16}$ This mechanism requires close proximity (essentially van der Waals contact) between donor and acceptor molecules for efficient energy transfer. This may explain why the simple presence of zeaxanthin in the membrane does not nec sssarily result in radiationless energy dissipation. ${ }^{1}$ Although our data demonstrate conclusively the energetic feasibility of zeaxanthin quenching chlorophyll a fluorescence, structural factors may also place constraints on determining in which systems zeaxanthin will be an effective quencher of the absorbed photon energy not used for photosynthesis.

This work has been supported in the laboratory of H. A. F. by the National Institutes of Health (GM-30353), the Cooperative State Research Service, and the University of Connecticut Research Foundation. The work at Argonne National Laboratory was supported by the Division of Chemical Sciences, Office of Basic Energy Sciences, US DOE, undei contract W-31-109-ENG-38. 


\section{References}

1. Demmig-Adams, B. Biochim. Biophys. Acta 1020, 1-24 (1990).

2. Siefermann-Harms, D. Biochim. Biophys. Acta 811, 325-355 (1985).

3. Gilmore, A. M. \& Yamamoto, H. Y. Proc. Natl. Acad. Sci. USA. 89, 1899-1903 (1992).

4. Horton, P. \& Ruban, A. V. Photosynth. Res. 34, 375-385 (1992).

5. Yamamoto, H. Y. Pure Appl. Chem. 51, 639-648 (1972).

6. Cogdell, R. J. \& Frank, H. A. Biochim. Biophys. Acta 895, 63-79 (1987).

7. Snyder, R., Arvidson, E., Foote, C., Harrigan, L. \& Christensen, R. L. J. Am. Chem. Soc. 107, 4117-4122 (1985).

8. DeCoster, B., Christensen, R. L., Gebhard, R., Lugtenburg, J., Farhoosh, R. \& Frank, H. A. Biockim. Biophys. Acta 1102, 107-114 (1992).

9. Shreve, A. P., Trautman, J. K., Frank, H. A., Owens, T. G. and Albrecht, A. C. Biochim. Biophys. Acta 1058, 280-288 (1991)

10. Frank, H. A., Farhoosh, R., Gebhard, R., Lugtenburg, J., Gosztola, D. \& Wasielewski, M. R. Chem. Phys. Lett. 207, 88 (1993)

11. Englman, R. \& Jortner, J. Mol. Phys. 18,145-164 (1970)

12. Wasielewski, M. R., Johnson, D. G., Bradford, E. G. \& Kispert, L. D. J. Chem. Phys. 91, 6691-6697 (1989).

13. Thrash, R. J., Fang, H. L.-B. \& Leroi, G. E. J. Chem. Phys. 67, 5930-5933 (1977).

14. Owens, T. G., Shreve, A. P. \& Albrecht, A. C. in Research in Photosynthesis, Murata, N. (ed.) Vol. 1, pp. 179-186, Kluwer Academic, The Netherlands (1992)

15. Horton, P., Ruban, A. V., Rees, D., Pascal, A. A., Noctor, G. \& Young, A. J. FEBS Lett. 292, 1 -4 (1991).

16. Dexter, D. L. J. Chem. Phys. 21, 836-860 (1953). 
Figure legends

Fig. 1. Schematic representation of the xanthophyll cycle showing the de-epoxidation of violaxanthin to zeaxanthin and the epoxidation of zeaxanthin to violaxanthin. Both of these reactions occur by way of antheraxanthin as an intermediate.

Fig. 2. The decay of the transient absorbance excited at $420 \mathrm{~nm}$ and probed at $540 \mathrm{~nm}, 529$ $\mathrm{nm}$, and $512 \mathrm{~nm}$ for violaxanthin, antheraxanthin and zeaxanthin, respectively. These wavelengths correspond to the maxima of the $2^{1} A_{g} \rightarrow S_{n}$ absorption of each xanthophyll. $\tau$ is the ${ }^{1} \mathrm{~A}_{\mathrm{g}}$ state lifetime obtained by fitting the decay curves to single exponential rate expressions.

Fig. 3. Schematic representation of the "molecular gear shift" mechanism of violaxanthin-tochlorophyll a forward energy transfer (light-harvesting function) and chlorophyll a-tozeaxanthin reverse energy transfer (non-photochemical fluorescence quenching function) induced by the enzymatic xanthophyll cycle. 


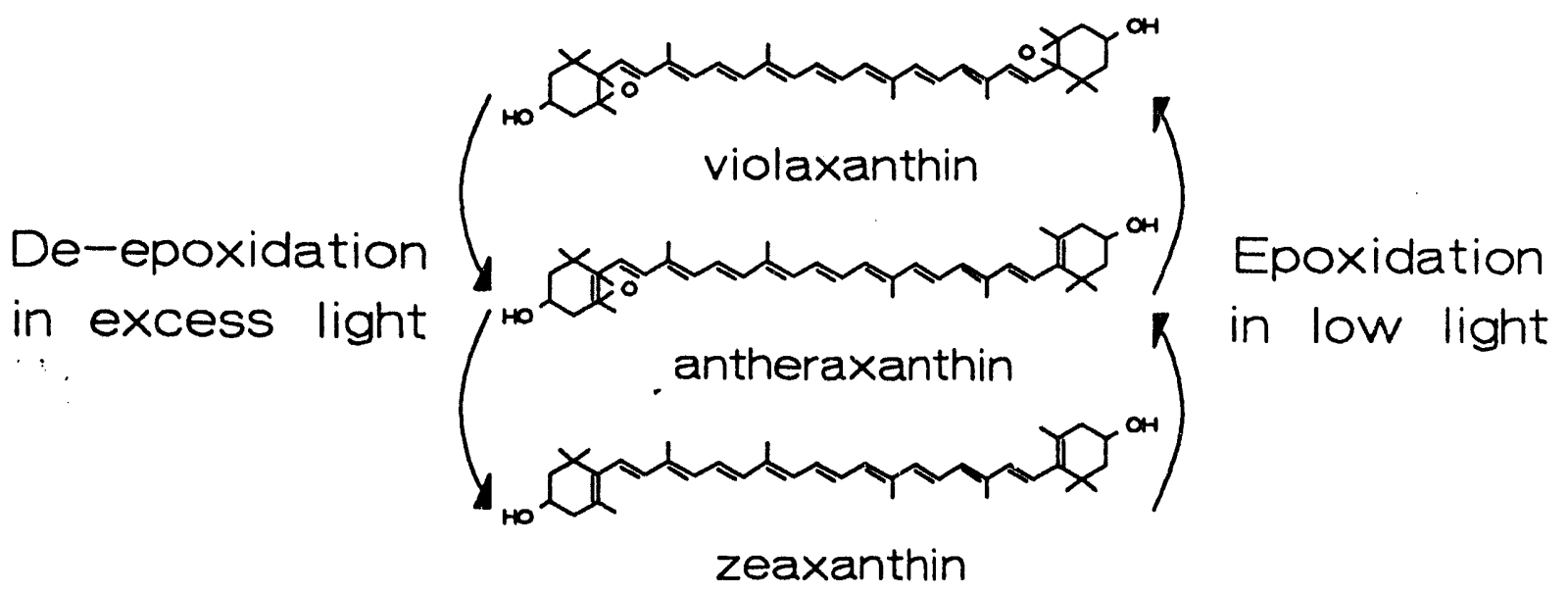



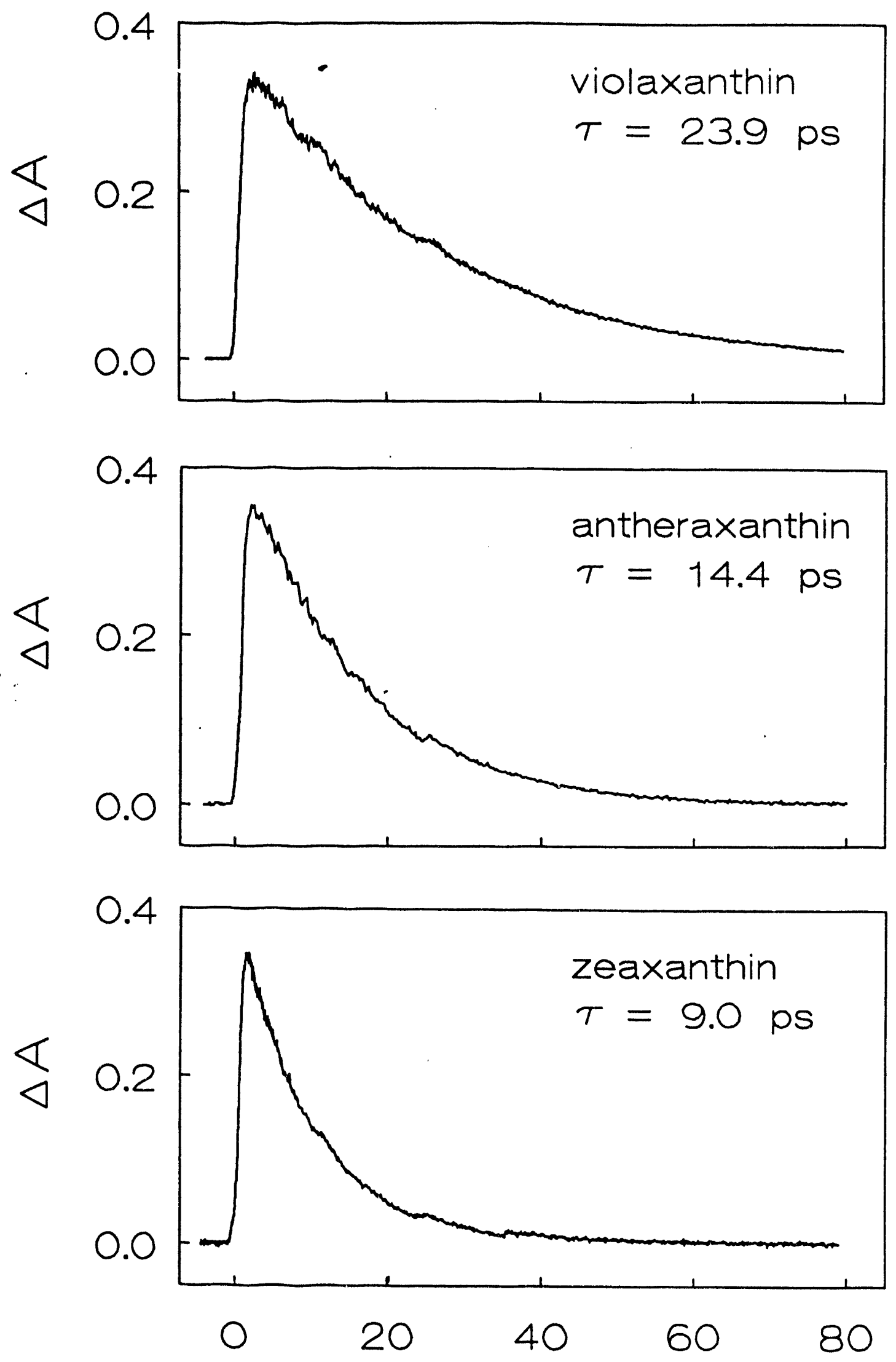

Time/ps 


\section{"Molecular Gear Shift" Mechanism Forward Energy Transfer}

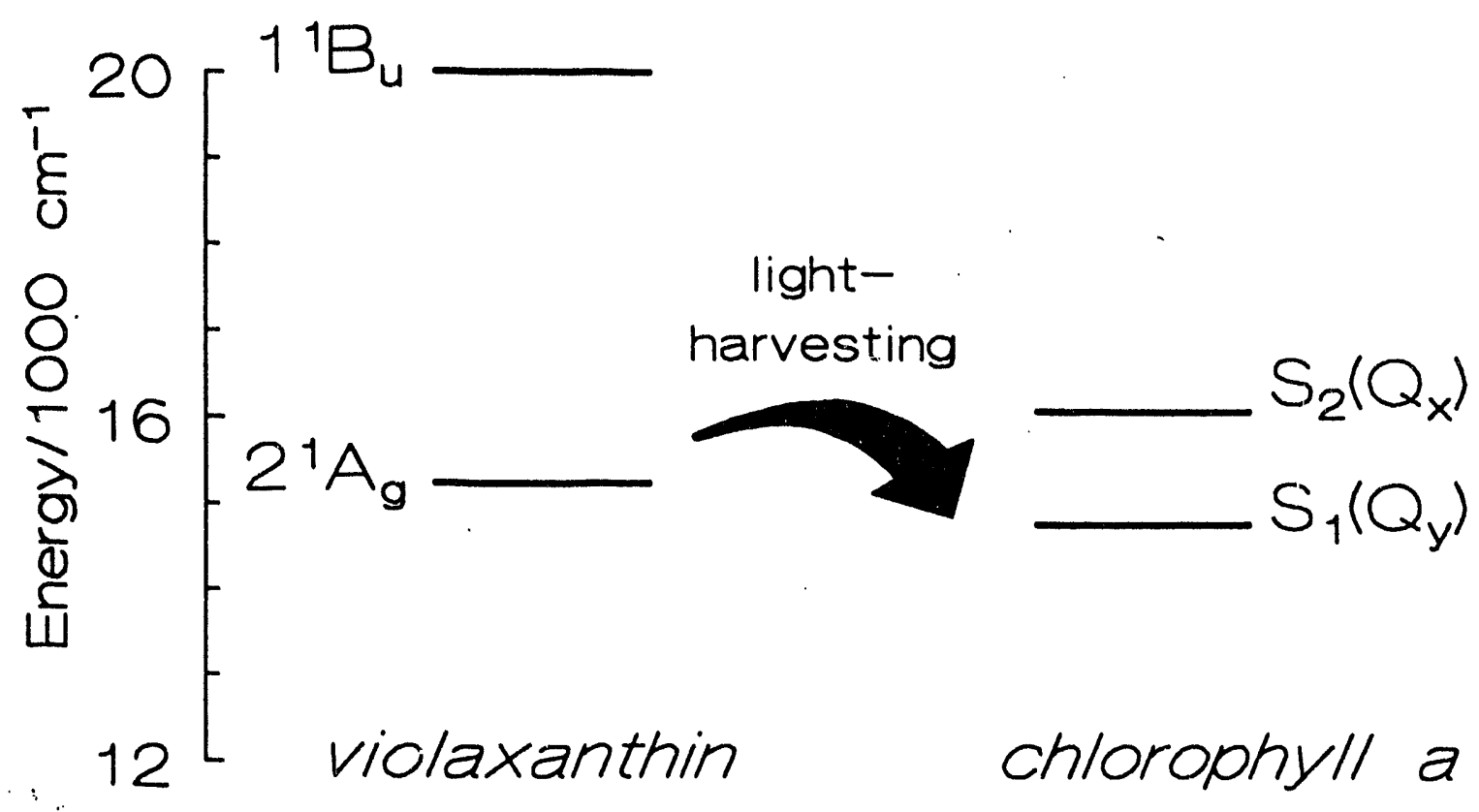

Reverse Energy Transfer

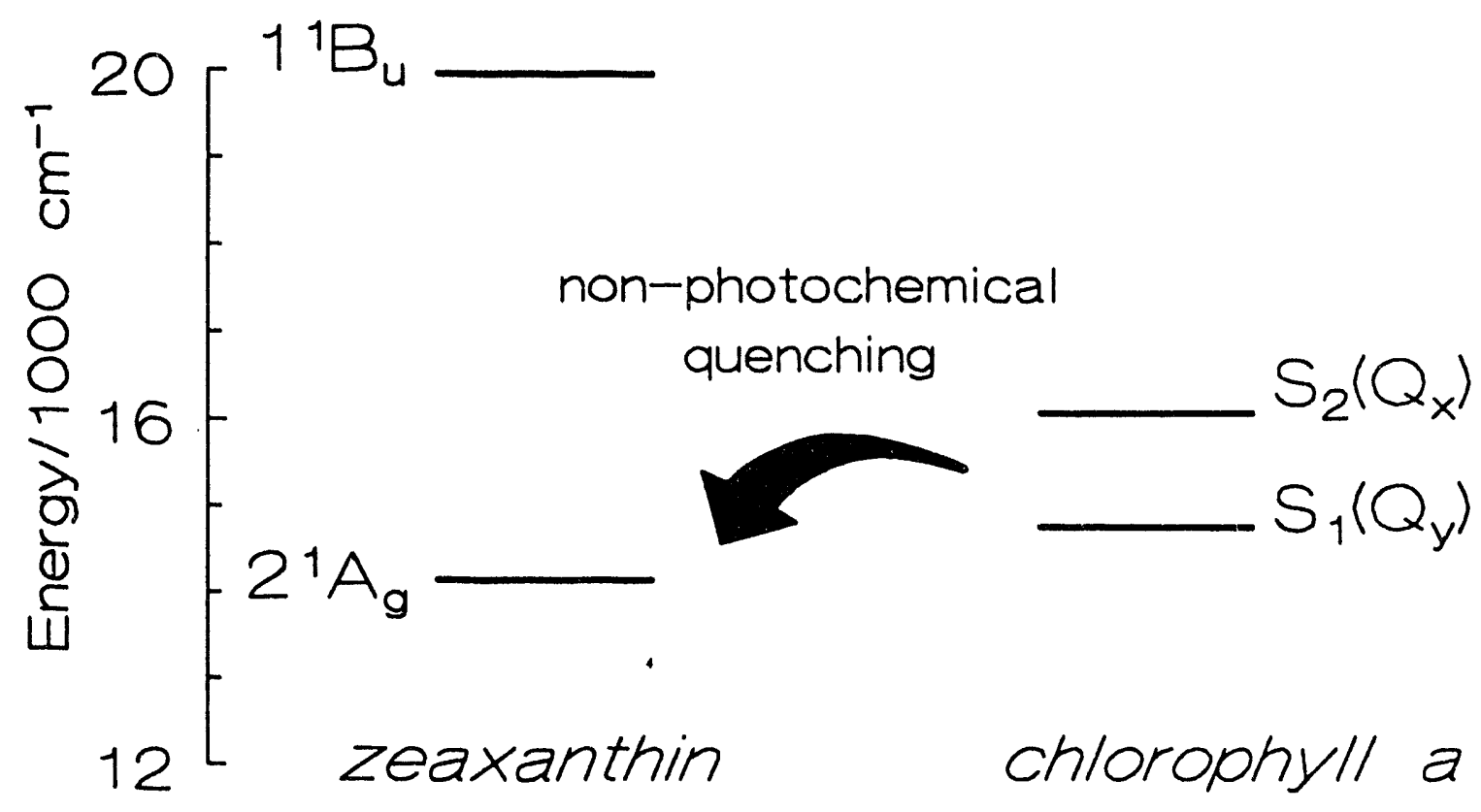



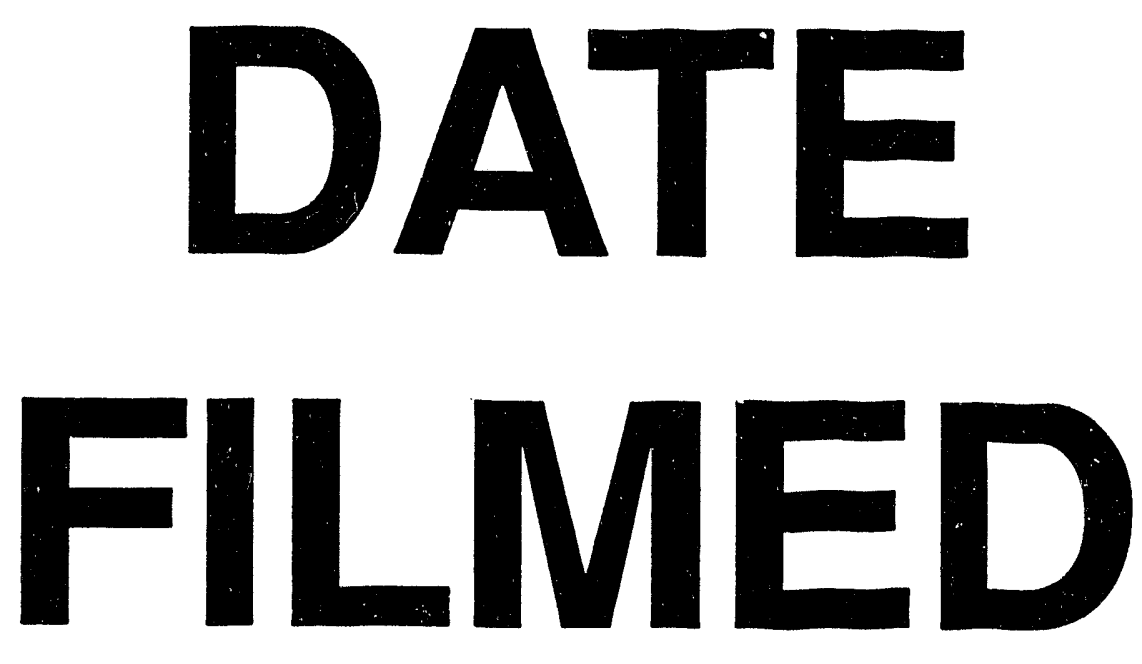

$10 / 17 / 94$
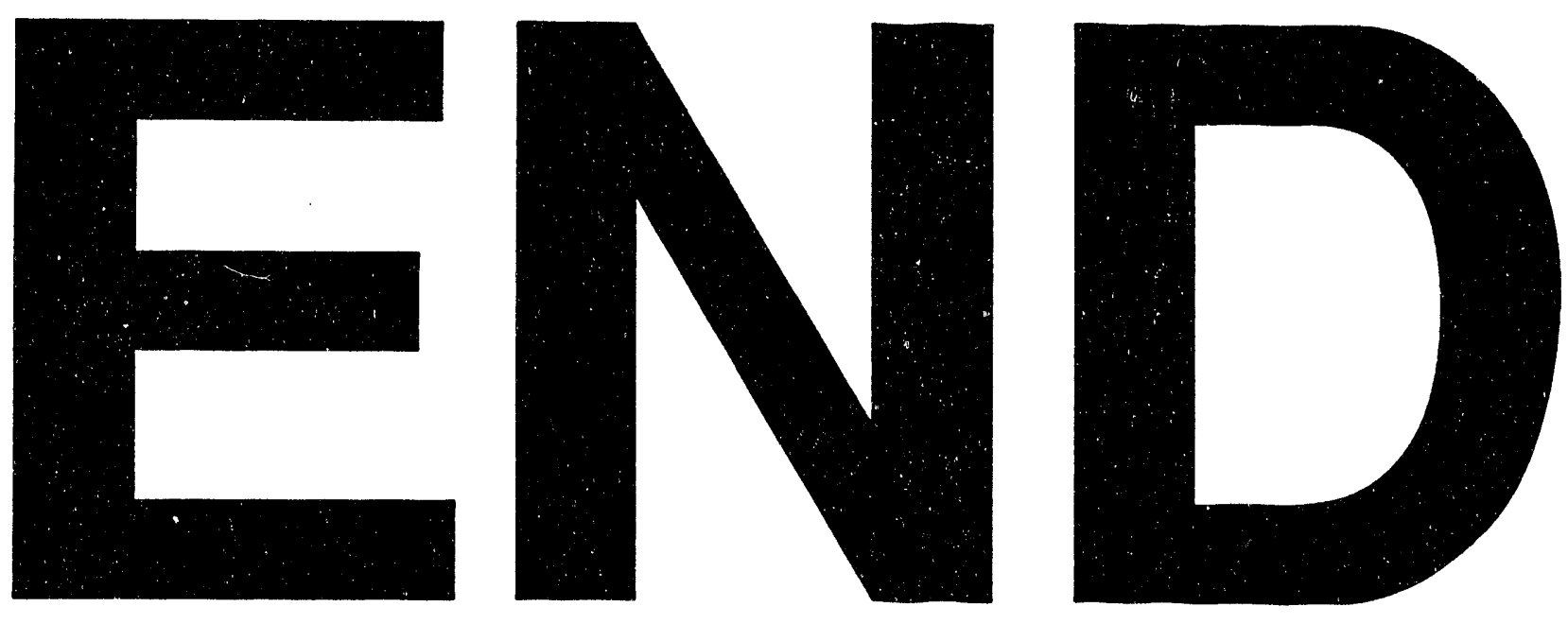